\title{
Self-generation of phase coherence in parallel Alfvén turbulence
}

\author{
Y. Nariyuki and T. Hada
}

E. S. S. T., Kyushu University, Japan

(Received April 5, 2005; Revised May 31, 2005; Accepted June 2, 2005)

\begin{abstract}
Nonlinear evolution of Alfvén turbulence is discussed within the framework of the derivative nonlinear Schroedinger equation (DNLS), a subset of the hall-MHD equation set, which includes quasi-parallel propagating right- and left-hand polarized Alfvén wave modes. By numerically time integrating the equation with periodic boundary conditions, we discuss relationship between generation of wave phase coherence and self-organization of the system due to birth of Alfvén solitons.
\end{abstract}

Key words: Alfvén waves, turbulence, complex system.

\section{Introduction}

Nonlinear evolution of Alfvén waves has been an attracting research subject in the context of magnetohydrodynamic (MHD) turbulence in cosmic plasmas, in particular, in the solar wind, where in situ measurement of electromagnetic as well as plasma variables is possible. The waves are considered to play essential roles in the heating and acceleration of energetic particles. In this letter, among various nonlinear processes triggered by the presence of finite amplitude Alfvén waves, we focus on generation of wave phase coherence by self-coupling of Alfvén waves caused by modulational instability (Mjølhus, 1974; Sakai and Sonnerup, 1983; Champeaux et al., 1999).

Recently, a method to quantitatively evaluate the degree of phase coherence for a given time series data has been proposed, by comparing structure functions of the given time series and its phase-shuffled as well as phase-equalized surrogates (Hada et al., 2003; Koga and Hada, 2003). By applying the method to magnetic field data obtained by Geotail spacecraft, it has been shown that the foreshock MHD turbulence is almost always phase correlated to a certain degree, and that the larger the turbulence level is, the stronger is the phase correlation, implying that the detected phase coherence is a consequence of nonlinear interaction among the MHD waves.

The finite phase coherence implies existence of local structures or steepened waves with sharp spatial gradient. The MHD turbulence with such localized waves/structures allows transport of energetic particles in a way qualitatively different from that described in quasi-linear type formulations, in which the random phase approximation is employed as one of the fundamental assumptions (Kuramitsu and Hada, 2000).

In this letter we discuss the relationship between generation of solitary waves and the wave coherence in detail, by examining numerically produced turbulence, using the

Copy right(c) The Society of Geomagnetism and Earth, Planetary and Space Sciences (SGEPSS); The Seismological Society of Japan; The Volcanological Society of Japan; The Geodetic Society of Japan; The Japanese Society for Planetary Sciences; TERRAPUB. derivative nonlinear Schrödinger (DNLS) equation.

\section{Alfvén Turbulence in the DNLS}

By applying a quasi-static approximation (i.e., variation of the plasma density is caused only by magnetic ponderomotive fluctuations) in which hydrodynamic nonlinearities and steepening are weak, Rogister (1971) derived a kinetic equation describing the long time evolution of the Alfvén waves. Essentially the same equation was subsequently obtained starting from two-fluid set of equations (Mjølhus, 1974; Mio et al., 1976; Spangler and Sheerin, 1982; Sakai and Sonnerup, 1983; Mjølhus and Hada, 1997). The fluid version, now known as the DNLS, reads

$$
\frac{\partial b}{\partial t}+\alpha \frac{\partial}{\partial x}\left(|b|^{2} b\right)+i \mu \frac{\partial^{2} b}{\partial x^{2}}=0
$$

where $x$ is the direction of propagation and the background magnetic field, $b=\left(B_{y}+i B_{z}\right) / B_{x}$ is the complex, transverse wave magnetic field normalized to $B_{x}$ (=const); $\alpha / C_{A}=C_{i}^{2} / 4\left(C_{i}^{2}-C_{s}^{2}\right)$, where $C_{A}, C_{i}, C_{s}$ are the Alfvén, intermediate, and sound speeds, respectively; and $2 \mu / C_{A}=c / \omega_{p i}$ is the ion inertia dispersion length. Time and space are normalized using the ion gyrofrequency and $C_{A}$.

The DNLS describes evolution of weakly nonlinear, quasi-parallel propagating, both right- and left-hand polarized Alfvén waves (or "magnetosonic" and "shear Alfvén" waves), which are nearly degenerate. Modulational instability is driven unstable for the left-hand polarized waves when $\beta=C_{s}^{2} / C_{A}^{2}<1$, and for the right-hand polarized waves when $\beta>1$ (Mjølhus, 1976; Spangler and Sheerin, 1982; Sakai and Sonnerup, 1983), although presence of resonant ions significantly alter the above conditions, especially for the case with $\beta>1$ (Rogister, 1971; Mjølhus and Wyller, 1988; Spangler, 1990; Medvedev et al., 1997). The DNLS is known to be integrable under various boundary conditions (Kaup and Newell, 1978; Kawata and Inoue, 1978; Kawata et al., 1980; Chen and Lam, 2004). In this letter, we restrict our discussion to the case with $\beta<<1$, since only in this case the use of the fluid version of the 
DNLS is justified, unless the ion to electron temperature ratio is extremely low.

By re-scaling of the variables, $t \rightarrow \mu t / \alpha^{2}$ and $x \rightarrow$ $\mu x / \alpha$, we can re-write (1) as

$$
\frac{\partial b}{\partial t}+\frac{\partial}{\partial x}\left(|b|^{2} b\right)+i \frac{\partial^{2} b}{\partial x^{2}}=0 .
$$

In Fig. 1 we show numerical time integration of (2) under periodic boundary conditions, where $|b|$ (envelope) is plotted in the phase space of time $(t)$ and space $(x)$. As initial conditions, finite amplitude, left-hand polarized, monochromatic Alfvén waves with the wave amplitude $b_{0}=0.4$ and the wave mode number $m=m_{0}=-11$ are given, superposed with a very small amplitude white noise with $<$ $\left|b_{\text {noise }}\right|^{2}>^{1 / 2}=10^{-5}$ within the range of $-256<m<256$, where the bracket denotes spatial average over the simulation system. In the above, the wave number $k$ is related to the mode number $m$ as $k=2 \pi m / L$, where the system size is $L=256$, in which there are 2048 grid points. We have used the convention that $m$ and $k$ positive (negative) representing the right- (left-) hand polarized waves. We have employed the rationalized Runge-Kutta scheme for time integration and the spectrum method for computing spatial derivatives.

Since we have monochrmoatic and circularly polarized waves as initial conditions, not much wave activities are evident in Fig. 1 at the beginning. However, starting from $t \sim 200$, modulation of the envelope becomes increasingly more evident, and around $t \sim 300$ a series of solitary waves is created. After that we observe a complex behavior of solitary waves: they appear, propagate, disappear, and interact with each other.

Corresponding time evolution of the power spectrum is shown in Fig. 2. During $0<t<\sim 200$, the parent wave energy $(m=-11)$ is gradually transferred to the side-band daughter waves (mainly, $m=-4$ and $m=-18$ ) through the modulational instability. Later on, increasingly more waves at different mode numbers are generated due to coupling among finite amplitude waves, and also due to the modulational instability of the daughter waves, as can be seen as widening of the power spectrum in the $m$-space. Around $t \sim 300$, the width of spectrum is maximized, corresponding to the appearance of the solitary waves. When the solitary waves disappear, the power spectrum becomes narrow again (the uncertainty principle, i.e., the width of the wave packet and that of the power spectrum are anticorrelated for wave evolution in a closed system). We note that solitary waves almost completely disappear at certain time intervals like $t \sim 900$, reminiscent of the presence of (near) recursion of the DNLS equation with periodic boundary conditions.

Let us write the Fourier transformed magnetic field $b_{k}=$ $\left|b_{k}\right| \exp i \phi_{k}$, and discuss the relation between behavior of solitary waves and correlation among wave phases $\phi_{k}$. First, we evaluate the wave phase coherence by computing the phase coherence index, $C_{\phi}$ (Hada et al., 2003; Koga and Hada, 2003): given a data containing the waves (in the present case, we use $B(x)=b(x, t)$ with fixed time), we make the phase randomized surrogate (PRS) and the phase correlated surrogate (PCS), by shuffling and making equal

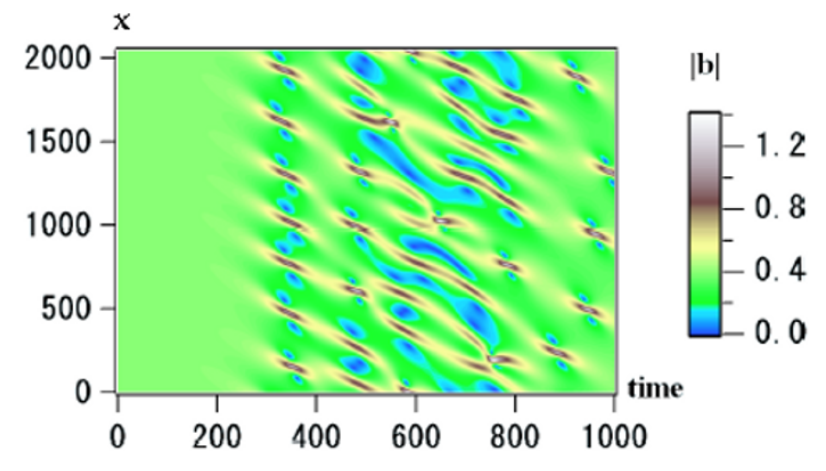

Fig. 1. Time evolution of envelope $|b|$ due to (2) with periodic boudary conditions. Initial conditions are given as a superposition of finite amplitude, left-hand polarized monochromatic waves, and very small amplitude white noise.

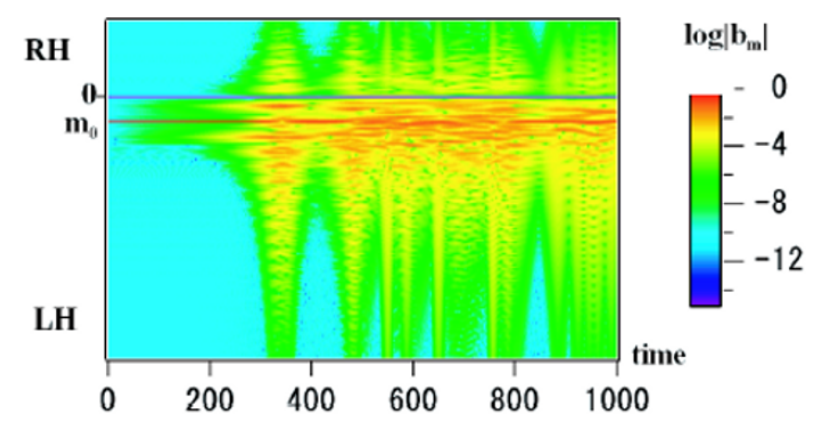

Fig. 2. Time evolution of power spectrum (in logarithmic scale), $\log \left|b_{m}\right|$, plotted in the phase space of the wave mode number $(m)$ and time. The parent mode is given initially at $m_{0}=-11$. Positive (negative) $m$ corresponds to right- (left-) hand polarized waves. The wave number is related to the wave mode number by $k=2 \pi m / L$, where $L=256$ is the system size.

the phases, respectively. Then we compute $C_{\phi}=\left(L_{P R S}-\right.$ $\left.L_{O R G}\right) /\left(L_{P R S}-L_{P C S}\right)$, where $L_{*}$ represents the first order structure function for the data $(*)$, e.g., $L_{O R G}=\sum_{x} \mid B(x+$ $\delta)-B(x) \mid$, where the coarsing scale $\delta$ was chosen to be the grid size. When $C_{\phi}$ is close to 0 , the wave phases are almost random, while when $C_{\phi}$ is close to unity, the wave phases are almost completely coherent. Figure 3 shows time evolution of $C_{\phi}$ for the run shown in Fig. 1. Apparently, the appearnce and disappearance of solitary wave trains correspond to the increase and decrease of $C_{\phi}$. This is easily understood since the solitary waveform may be produced by superposition of many waves with different wavelengths with equal phase.

Now we discuss how the phase coherence is generated in the DNLS. First we note that the nonlinearity of the DNLS equation is cubic, i.e., the basic nonlinear interaction is the four wave resonance (interaction among a quartet of wave modes). Namely, we have the resonance relation among the wave numbers,

$$
k_{1}+k_{2}=k_{3}+k_{4}
$$

and a similar relation should be held for the wave frequencies as well. Let us define the relative phase among the four waves as

$$
\theta(\mathbf{k}) \equiv \phi_{k 1}+\phi_{k 2}-\phi_{k 3}-\phi_{k 4}
$$




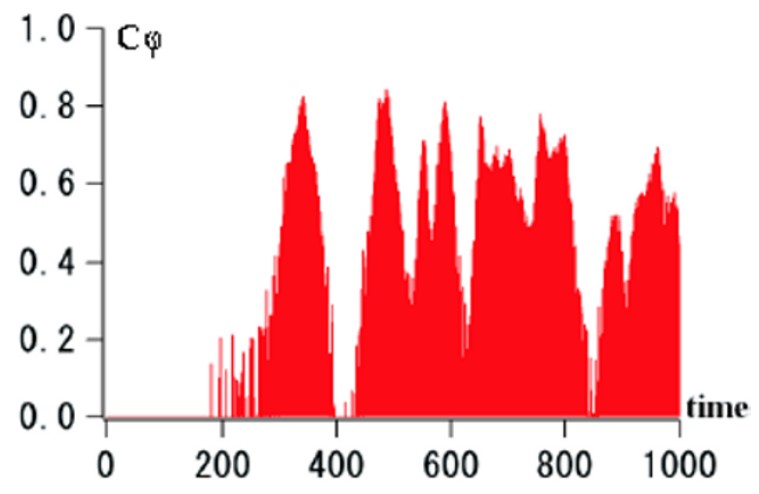

Fig. 3. Time evolution of $C_{\phi}$. When $C_{\phi} \sim 0$, the wave phases are almost random, while $C_{\phi} \sim 1$ represents that the wave phases are almost completely correlated.

where $\mathbf{k}=\left\{k_{1}, k_{2}, k_{3}, k_{4}\right\}$ represents the quartets of wave modes in resonance. Small temporal change of $\theta_{k}$ implies that the phase correlation between the four waves is strong (locked). From (2) and (4), we have

$$
\frac{d}{d t}\left(\frac{\left|b_{k 1}\right|^{2}}{2}\right)=k_{1} \sum\left|b_{k 1}\right|\left|b_{k 2}\right|\left|b_{k 3}\right|\left|b_{k 4}\right| \sin \theta(\mathbf{k})
$$

where the summation is to be taken over all the combinations of $\left(k_{2}, k_{3}, k_{4}\right)$ which satisfy (3). This equation indicates that the evolution of wave energy of a certain mode is determined by 'energy flow' exchanged between the quartets,

$$
F(\mathbf{k}) \equiv k_{1}\left|b_{k 1}\right|\left|b_{k 2}\right|\left|b_{k 3}\right|\left|b_{k 4}\right| \sin \theta(\mathbf{k})
$$

We note that the direction of the energy flow is determined by the signs of $\sin \theta(\mathbf{k})$ and $k_{1}$.

Figure 4 shows the relation between $F(\mathbf{k})$ and $|\theta(\mathbf{k})|$. Here it is seen that the exchange of wave action among the four wave modes is enhanced (reduced) when the relative phase is close to constant (varies rapidly) in time. The same tendency is seen in all the quartets in resonance. From this result, we interpret the nonlinear time evolution of Figs. 1-3 in detail: Around $t \sim 200$, due to the modulational instability driven by the finite amplitude parent wave, a pair of side band waves (daughter waves) appears. At this stage, the phase correlation is generated only within a small number of resonant quartets. The growing daughter waves are restricted within $k<0$, because of the derivative nonlinear term in (2) (Mio et al., 1976). In other words, if a quartet includes some right-hand polarized wave modes, this quartet is stable, and the exchange of energy among them stays only at a fluctuation level. After the daughter wave modes grow sufficiently large around $t \sim 300$, quartets of waves which include the $k>0$ modes become unstable also. Because of this, wave modes near $k \sim 0$ are amplified. This broading of the power spectrum (Fig. 2) corresponds not only to generation of solitary waves (Fig. 1), but also to the generation of the phase coherence (Fig. 3): large number of quartets have significant nonlinear interaction and generate the wave phase coherence.

From Fig. 2 we see that the parent wave mode $\left(k=k_{0}\right)$ remains to be the dominant one throughout the simulation run. Therefore, among the many quartets of wave

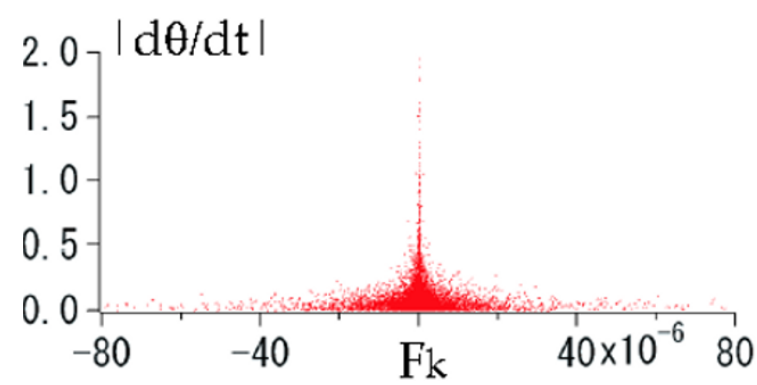

Fig. 4. Relation between $F(\mathbf{k})$ and $|\theta(\mathbf{k})|$. The exchange of wave energy (wave action) between the sites is enhanced (reduced) when the relative phase is almost constant (vary rapidly) in time. The unit of the vertical axis is degrees per unit time.

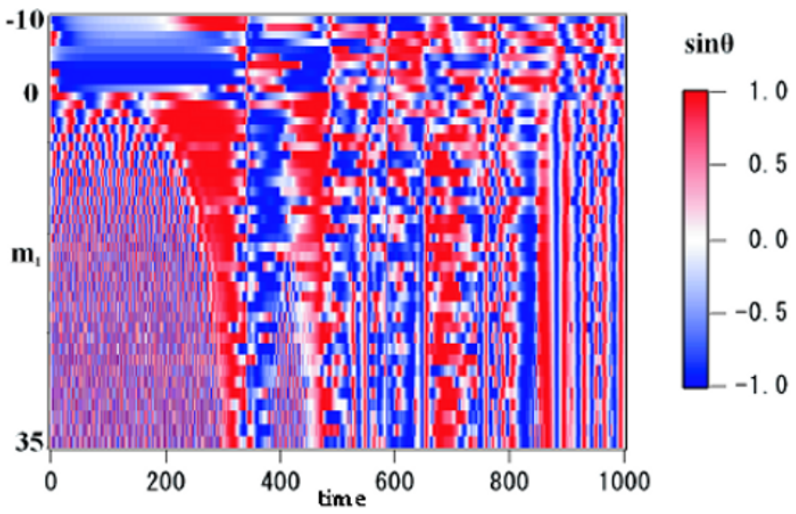

Fig. 5. Evolution of $\sin \theta(\mathbf{k})$, plotted in the phase space of the wave mode number $\left(m_{1}\right)$ and time.

modes in resonance $\left(k_{1}, k_{2}, k_{3}, k_{4}\right)$ with $k_{3}+k_{4}=k_{1}+k_{2}$, the most dominant quartet (which makes (6) largest) is $\left(k_{1}, k_{2}, k_{0}, k_{0}\right)$, which satisfies the resonance condition,

$$
k_{0}+k_{0}=k_{1}+k_{2} \text {. }
$$

In Fig. 5 we have plotted the value of $\sin \theta(\mathbf{k})$ in the phase space of $m_{1}$ and time, with $k_{3}=k_{4}=k_{0}$ and $k_{2}$ determined by (7). (The wave number $k$ and the wave mode number $m$ are related by $k=2 \pi m / L$, with the system size $L=256$.) The plot shows the direction of energy flow between the parent wave and daughter waves. For example, associated with the first formation of solitary wave rows $(t \sim 200-$ $350), \sin \theta(\mathbf{k})<0$ for $k_{1}<0$ and $\sin \theta(\mathbf{k})>0$ for $k_{1}>0$. Namely, in both regimes of $k_{1}>0$ and $k_{1}<0$, the energy flow defined in (6) is positive, so that the energy is transferred from the parent wave to daughter waves. During the time interval when solitary waves disappear $(t \sim 350-$ 400), we observe the opposite, suggesting that the energy flows back from the daughter to parent waves.

\section{Discussion}

In this letter we discussed generation of the phase correlation in Alfvén turbulence using the DNLS equation, which models nonlinear evolution of weakly nonlinear, quasi-parallel propagating Alfvén waves in either right- or left-hand polarization. Long time evolution of finite am- 
plitude circularly polarized Alfvén waves leads to generation of a series of solitary waves due to the modulational instability, followed by their complex interaction. Generation of the solitary waves corresponds to broadening of the wave power spectrum and enhancement of the phase coherence index. By examining the energy flow among quartet of wave modes, we have identified that the parent wave mode continues to be the main source for providing energy to daughter wave modes within the time scale of our simulation run. Our main conclusion of this letter is in Fig. 4: the relative phase among the quartet of waves is strongly related to the energy flow among the waves. When the wave modes exchange energy, the phases are 'locked', and when there is no exchange of energy, the phases can slip freely. We have made simulations runs varying initial conditions, and also after modifying the equation by adding damping and driving terms (Spangler, 1986; Ghosh and Papadopoulos, 1987; Hada, 1992). Essentially the same results are obtained regarding the relationship between appearance of the solitary waves, exchange of energy among the wave modes, and the enhancement of the wave phase coherence.

Finally, we make a remark on energetic particle transport in the turbulence with phase coherence. We see that solitary waves are created due to strong modulation of the parent Alfvén wave envelope. Since such solitary waves have broad power spectrum in the $k$ space, particles with wide range of energy can resonate with them. However, this is not the more important side of the wave-particle interaction, from the viewpoint of the phase coherence - rather, it is in the fact that large amount of electromagnetic field and plasma energy is concentrated within the solitary wave (phase coherent wave/structure), so that it can influence particle motion via strong and correlated impulse force rather than via sequence of random forces which last for a long time. For example, let us consider pitch angle diffusion due to the turbulence consisting of superposition of parallel, circularly polarized Alfvén waves (slab model). Within this model, it is well known that the particles cannot diffuse across 90 degrees pitch angle, within the framework of the quasi-linear diffusion, simply because there are no waves which can resonate with particles without parallel velocities. However, the mirror force can reflect the particles quite easily, if there exist solitary waves with a finite amplitude variation of the total magnetic field. Furthermore, in a presence of many of these solitary waves, individual particle trajectory may essentially be given as combination of quasi-ballistic motion between the solitary waves and trapping by one of the solitary waves. Fermi type acceleration is possible (Kuramitsu and Hada, 2000), and furthermore, depending on relative dominance between the ballistic and trapped trajectories, ensemble of these particles may appear as either super- or sub-diffusive (Zimbardo et al., 2000; Carreras et al., 2001). In order to properly describe time evolution of the ensemble, one has to invoke the fractal diffusion formalism (Metzler and Nonnenmacher, 1998; delCastillo-Negrete et al., 2004).
Acknowledgments. We thank valuable discussions with Dr. D. Koga, Dr. S. Matsukiyo, Dr. V. Munoz, and Mr. K. Seki.

\section{References}

Carreras, B. A., V. E. Lynch, and G. M. Zaslavsky, Anomalous diffusion and exit time distribution of particle tracers in plasma turbulence model, Phys. Plasmas, 8(12), 5096-5103, 2001.

del-Castillo-Negrete, D., B. A. Carreras, and V. E. Lynch, Fractional diffusion in plasma turbulence, Phys. Plasmas, 11(8), 3854-3864, 2004.

Champeaux, S. et al., Remarks on the parallel propagating of smallamplitude dispersive Alfvén waves, Nonl. Proc. in Geophys., 6, 169178, 1999.

Chen, X. J. and W. K. Lam, Inverse scattaring transform for the derivative nonlinear Schrodinger equations with nonvanishing boundary conditions, Phys. Rev. E, 69, art. no. 066604, 2004.

Ghosh, S. and K. Papadopoulos, The onset of Alfvénic turbulence, Phys. Fluids., 30(5), 1371-1387, 1987.

Hada, T., Nonlinear evolution of Alfvén waves in space plasmas, in Nonlinear Processes in Physics, edited by A. S. Fokas, D. J. Kaup, A. C. Newell, and V. E. Zhakarov, pp. 169-174, Springer-Verlag, 1992.

Hada, T., D. Koga, and E. Yamamoto, Phase coherence of MHD waves in the solar wind, Space Sci. Rev., 107(1-2), 463-466, 2003.

Kaup, D. J. and A. C. Newell, An exact solution for a derivative nonlinear Schrodinger equation, J. Math. Phys, 19, 798-801, 1978.

Kawata, T. and $H$. Inoue, Exact solution of the derivative nonlinear Schrödinger equation under the nonvanishing conditions. J. Phys. Soc. Japan, 44, 1968-1976, 1978.

Kawata, T., J. -I. Sakai, and N. Kobayashi, Inverse method for the mixed nonlinear Schrödinger equation and soliton solutions. J. Phys. Soc. Japan, 48, 1371-1379, 1980.

Koga, D. and T. Hada, Phase coherence of foreshock MHD waves: wavelet analysis, Space. Sci. Rev, 107, 495-498, 2003.

Kuramitsu, Y. and T. Hada, Acceleration of charged particles by large amplitude MHD waves: effect of wave spatial correlation, Geophys. Res. Lett., 27(5), 629-632, 2000.

Medvedev, M. D., P. H. Diamond, V. I. Shevchenko, and V. L. Galinsky, Dissipative dynamics of collisionless nonlinear Alfvén wave trains, Phys. Rev. Lett., 78(26), 4934-4937, 1997.

Metzler, R. and T. F. Nonnenmacher, Fractional diffusion, waiting-time distributions, and Cattaneo-type equations, Phys. Rev. E, 57(6), 64096414, 1998.

Mio, K., T. Ogino, K. Minami, and S. Takeda, Modified nonlinear Schroedinger equation for Alfvén waves propagating along the magnetic field in cold plasmas. J. Phys. Soc. Japan, 41, 265-271, 1976.

Mjølhus, E., Application off the reductive perturbation method to long hydromagnetic waves parallel to the magnetic field in a cold plasma, Report no. 48, Department of applied mathematics, Univ. Bergen, Norway, 1974.

Mjølhus, E., On the modulational instability of hydromagnetic waves parallel to the magnetic field, J. Plasma. Phys., 16, 321-334, 1976.

Mjølhus, E. and T. Hada, Soliton theory of quasi-parallel Alfvén waves, in Nonlinear Waves and Chaos in Space Plasmas, edited by T. Hada and H. Matsumoto, pp. 121-169, Terrapub, Tokyo, 1997.

Mjølhus, E. and J. Wyller, Nonlinear Alfvén waves in a finite beta plasma, J. Plasma Phys., 40, 299-318, 1988.

Rogister, A., Parallel propagation of nonlinear low-frequency waves in high- $\beta$ plasma, Phys. Fluids, 14, 2733-2739, 1971.

Sakai, J. -I. and B. Sonnerup, Modulational instability of finite amplitude dispersive Alfvén waves, J. Geophys. Res, 88, 9069-9078, 1983.

Spangler, S. R., The evolution of nonlinear Alfvén waves subject to growth and damping, Phys. Fluids, 29(8), 2535-2547, 1986.

Spangler, S. R., Kinetic effects on Alfvén-wave nonlinearity, 2. The modified nonlinear-wave equation, Phys. Fluids, B2(2), 407-418, 1990.

Spangler, S. R. and J. P. Sheerin, Properties of Alfvén solitons in a finitebeta plasma, J. Plasma Phys., 27, 193-198, 1982.

Zimbardo, G., A. Greco, and P. Veltri, Superballistic transport in tearing driven magnetic turbulence, Phys. Plasmas, 7(4), 1071-1074, 2000.

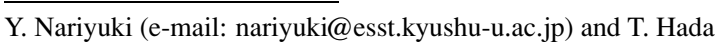

